\title{
Combined Use of Intraoperative Magnetic Resonance Imaging and Neuronavigation for Microsurgical Approach of Intractable Epilepsy:a Systematic Review and Meta-analysis
}

\author{
Constantin Tuleasca ( $\sim$ constantin.tuleasca@gmail.com ) \\ University of Lausanne \\ Iulia Peciu-Florianu \\ Centre Hospitalier Régional et Universitaire de Lille \\ Maxime Chochoi \\ Centre Hospitalier Régional et Universitaire de Lille \\ Marc Levivier \\ University of Lausanne \\ Benoit Derre \\ Centre Hospitalier Régional et Universitaire de Lille \\ Philippe Derambure Derambure \\ Centre Hospitalier Régional et Universitaire de Lille \\ Nicolas Reyns \\ Centre Hospitalier Régional et Universitaire de Lille
}

Research Article

Keywords: epilepsy, intraoperative MRI, microsurgery, resection, Engel

Posted Date: January 17th, 2022

DOI: https://doi.org/10.21203/rs.3.rs-1206251/v1

License: @ (i) This work is licensed under a Creative Commons Attribution 4.0 International License. Read Full License 


\section{Abstract}

\section{Introduction}

Epilepsy is a major feature of multiple types of lesional epilepsy (LE) and non-lesional (NLE). However, there is an important heterogeneity in the use of such nomenclature. A favorable outcome is correlated to an identifiable cause and the extent of resection. Here, we evaluate the use of intraoperative MRI (iMRI) combined with neuronavigation, in improving the complete and/or adequate resection rates in patients with intractable epilepsy of various etiologies. We further discuss whether this results in better clinical outcomes.

\section{Material and methods}

A medical librarian performed a comprehensive review of literature, searching the Medline $₫$, Embase ${ }^{\circledR}$, Index Medicus $®$ and Cochrane $₫$ databases. Two reviewers independently applied following inclusion criteria: reports of at least two cases of any age undergoing microsurgical resection for epilepsy surgery, the use of iMRI as mandatory for intraoperative assessment, evaluation of LE versus NLE, describing if second resection after iMRI was necessary, detailing the extent of resection, qualitative reports of seizure outcome (Engel class).

\section{Results}

We report 15 studies, encompassing 867 patients, with various types of LE and NLE. Most common surgical indications for the use of iMRI in LE were dysembrioplastic neuroepithelial tumor (DNET) and gangliogliomas, cavernomas, hypothalamic hamartomas, or primary brain tumors. For NLE these were sclerosis, dysplasia, ischemia and gliosis, which are further separately detailed in a supplementary material. With the use of iMRI, the rate of complete or adequate resection significantly increased from 74.8\% (95\% confidence interval (Cl) $67.3-82.3 \%$ ) to $97.6 \%$ (95\% confidence interval (Cl) $95.8-99.3 \%$ ). Hundred and twenty-six cases out of 820 underwent additional resection after iMRI, for an overall rate of $24.4 \%$ (range 16.9-31.8). This was translated in an overall clinical benefit with $77.1 \%$ (95\% Cl 71-83.2\%) Engel class I at last follow-up $(\mathrm{p}<0.001)$.

\section{Discussion}

Intraoperative MRI might improve the extent of resection in LE and NLE. This might translate into a favorable clinical outcome (Engel class I as high as 77\%). This is fairly comparable to what one expects in temporal lobe epilepsy surgery. We suggest that iMRI is beneficial when proposed in selected cases of intractable epilepsy. However, the reader should take into account that such data are not provided by randomized controlled trials and that such conclusions should be carefully interpreted.

\section{Introduction}

Epilepsy is a clinical concept that encompasses a heterogeneous group of syndromes, with different etiologies, clinical impact, severity and therapeutic options(1). Epilepsy has been defined conceptually in 2005 as a brain disorder characterized by an enduring predisposition to generate epileptic seizures(2). In a recent systematic review, the point prevalence was 6.38 per 1000 persons, with an annual cumulative incidence of 67.77 per 100.000 persons(3).

Current understanding of natural history, neurobiology and pathophysiology had progressively evolved, further leading to a change in therapeutic approaches(4). Moreover, the understanding of prognostic factors has improved, generating a "prognostic group specific" management that could be explored. In this context, effective treatments may be used in a more rational and targeted fashion(4).

The surgical management is based on type of epilepsy, anatomical location, patient's preference, as well as surgeon's expertise(1). Current surgical procedures include resection, disconnection and neural modulation(5). Seizure control rates in patients with focal epilepsy benefiting from surgery ranges between 33$90 \%(5-10)$. The former has been improved in recent trials $(9,11)$ and varies between $80-90 \%$ (Engel class I or II(12)) in single and identified MRI structural abnormality (lesional epilepsy, LE) as compared to $36 \%$ in non-lesional epilepsy (NLE)(13). Factors associated with good outcome include single unilateral MRI abnormality, localized ipsilateral ictal and interictal epileptiform activity and unilateral hippocampal sclerosis(8, 14). Poor outcome relates to absence of MRI abnormality or multiple MRI abnormalities, bilateral atrophy, suspected cortical dysplasia and non-localizing electroencephalographic (EEG) results(8).

The improvement in neuroimaging and microsurgical techniques has allowed a more specific localization of epileptic foci as well as tailored resections(15, 16). The introduction of frameless stereotaxy further permitted precise craniotomy placement and optimization of the surgical trajectory, avoiding critical structures during intraoperative dissection(17). However, tissue dissection, brain retraction, cerebrospinal fluid loss and gravity often result in brain shift, nullifying preoperative images.

During the past two decades, the development of intraoperative MRI (iMRI) has allowed real-time updating of intracranial neuroanatomy and surgical advancement. Coupled with neuronavigation, it provides real-time valuable information, allowing even more tailored epileptic foci resection, while decreasing morbidity and mortality. However, the use of iMRI in epilepsy varies from center to center in terms of indications, extent of resection, number of controls during surgery in iMRi, surgical outcome etc. Moreover, the benefit of iMRI in epilepsy surgery has not provided level I evidence in terms of safety and efficacy.

Here, we aim at providing a systematic report of studies using iMRI for drug-resistant epilepsy surgery, summarizing the results to date. We further detail its use in both LE and NLE. Our main goal is to provide clinicians with scientifically valid and comprehensible evidence, and to provide estimates of surgical success before and after the use of iMRI in terms of the extent of resection. We further evaluate the clinical outcome at last follow-up in terms of Engel class.

\section{Material And Methods}




\section{Data sources}

A medical librarian performed a comprehensive review of literature, searching the Medline ${ }^{\circledR}$, Embase $\AA$, Index Medicus $\AA$ and Cochrane ${ }^{\circledR}$ databases. Moreover, potential studies from systematic reviews were revised in full text to identify relevant articles. We additionally consulted experts and searched for bibliographies on the same topic. In order to benefit from a homogenous dataset, we focused on full-length articles published in English during a 20-years period of time, from January 1999 to December 2019.

\section{Study selection, classification and data screening}

The databases were queried using the following word combinations (mesh terms) in the "title" or "title/abstract" item: ("epilepsy" AND "intraoperative" AND "MRI"), or combination of these words, including ("epilepsy" AND "intraoperative" AND "MRI") etc. We also used the Google Scholar and Google search engine to expand our list of studies, including abstracts, but encompassed in the final analysis only peer-reviewed papers.

Two reviewers (CT, IP) independently applied the following inclusion criteria: a). reports of >=2 cases of any age undergoing microsurgical resection for epilepsy surgery; b). the use of iMRI as mandatory for intraoperative assessment, c). evaluation of LE versus NLE, d). describing if a second evaluation after iMRI was necessary, e). detailing the extent of resection, f). qualitative reports of seizure outcome in terms of Engel class; g). all types of MRI field were included. All included studies reported consistent definitions for both LE and NLE. Moreover, one has to take into account the progressive refinement over time of the MRI techniques and the associated continuous increase of the MR fields in the operative room.

Were excluded studies in other languages than English.

Potentially relevant articles were selected for a full-text screening evaluation, which was independently performed by 3 investigators (CT, IP, NR). Discrepancies were resolved by the corresponding and senior authors (CT, NR).

\section{Data extraction}

Were extracted 15 studies $(1,18-31)$, which included 867 patients (Tables 1 and 2). A flow-chart describing the study selection was created and is displayed in Figure 1. Study characteristics, publication year, sample size, follow-up, anatomical location, LE versus NLE, complete/adequate resection, and second evaluation after iMRI as well as Engel outcome were extracted. 
Table 1

Summary of series: basic demographic data

\begin{tabular}{|c|c|c|c|c|c|c|c|c|c|c|}
\hline $\begin{array}{l}\text { Author } \\
\text { (year) }\end{array}$ & $\begin{array}{l}\text { Center } \\
\text { (Country) }\end{array}$ & $\begin{array}{l}\text { MR } \\
\text { power } \\
(\mathrm{T})\end{array}$ & Number & $\begin{array}{l}\text { Pediatric/ } \\
\text { Adult }\end{array}$ & $\begin{array}{l}\text { Demographic } \\
\text { details (age, } \\
\text { years) }\end{array}$ & $\begin{array}{l}\text { Follow- } \\
\text { up }\end{array}$ & $\begin{array}{l}\text { Type of } \\
\text { epilepsy }\end{array}$ & Location & $\begin{array}{l}\text { Lesional/ } \\
\text { nonlesional }\end{array}$ & Callosotom \\
\hline $\begin{array}{l}\text { Buchfelder } \\
(2000)\end{array}$ & $\begin{array}{l}\text { Erlangen } \\
\text { (Germany) }\end{array}$ & 0.2 & 61 & $\begin{array}{l}\text { Both (not } \\
\text { specified) }\end{array}$ & $\begin{array}{l}2-60 \\
32.3 \pm 11.9\end{array}$ & NA & PharmacoR & $\begin{array}{l}\text { Temporal } \\
\text { resection \& } \\
\text { callosotomy }\end{array}$ & $29 / 28$ & $n=4$ \\
\hline $\begin{array}{l}\text { Buchfelder } \\
(2002)\end{array}$ & $\begin{array}{l}\text { Erlangen } \\
\text { (Germany) }\end{array}$ & 0.2 & 58 & $5 / 53$ & $\begin{array}{l}2-60 \\
33 \pm 12\end{array}$ & $\begin{array}{l}\text { mean: } \\
15.5 \\
\text { lesional, } \\
10.7 \\
\text { non- } \\
\text { lesional }\end{array}$ & PharmacoR & $\begin{array}{l}\text { Temporal } \\
\text { resection }\end{array}$ & $29 / 28$ & No \\
\hline $\begin{array}{l}\text { Kaibara } \\
(2002)\end{array}$ & $\begin{array}{l}\text { Calgary } \\
\text { (Canada) }\end{array}$ & 1.5 & 14 & $0 / 14$ & $\begin{array}{l}23-55 \\
36 \pm 10\end{array}$ & $\begin{array}{l}\text { median: } \\
17 \pm 3.4\end{array}$ & PharmacoR & $\begin{array}{l}\text { Temporal } \\
\text { resection }\end{array}$ & $0 / 14$ & No \\
\hline $\begin{array}{l}\text { Schwarz } \\
(2002)\end{array}$ & $\begin{array}{l}\text { (New Jersey, } \\
\text { USA) }\end{array}$ & 0.12 & 5 & $3 / 2$ & $7-65$ & $\begin{array}{l}\text { mean: } \\
9.2\end{array}$ & PharmacoR & $\begin{array}{l}\text { Temporal } \\
\text { resection }\end{array}$ & $1 / 4$ & No \\
\hline $\begin{array}{l}\text { Walker } \\
(2002)\end{array}$ & $\begin{array}{l}\text { Boston } \\
\text { (USA), } \\
\begin{array}{l}\text { Brisbane } \\
\text { (Australia) }\end{array}\end{array}$ & & 13 & $2 / 13$ & $\begin{array}{l}26.1 \text { years } \\
\text { (range } 5 \pm 40 \text { ) }\end{array}$ & $\begin{array}{l}\text { mean } \\
22.1 \\
\text { months } \\
\text { (range } \\
2 \pm 48 \text { ) }\end{array}$ & PharmacoR & $\begin{array}{l}\text { Temporal and } \\
\text { other }\end{array}$ & $0 / 11$ & No \\
\hline $\begin{array}{l}\text { Kelly } \\
(2005)\end{array}$ & $\begin{array}{l}\text { Calgary } \\
\text { (Canada) }\end{array}$ & 1.5 & 70 & $11 / 59$ & $33 \pm 13$ & $>6$ & PharmacoR & $\begin{array}{l}\text { Temporal and } \\
\text { other }\end{array}$ & $9 / 58$ & $n=3$ \\
\hline $\begin{array}{l}\text { Sun } \\
(2011)\end{array}$ & $\begin{array}{l}\text { Beijing } \\
\text { (China) }\end{array}$ & 1.5 & 36 & $4 / 32$ & $\begin{array}{l}10-56 ; \\
32.78 \pm \\
12.92\end{array}$ & $\begin{array}{l}\text { mean: } \\
12.6\end{array}$ & $\begin{array}{l}9 / 36 \\
\text { PharmacoR }\end{array}$ & $\begin{array}{l}\text { Eloquent } \\
\text { areas, mixed }\end{array}$ & $36 / 0$ & No \\
\hline $\begin{array}{l}\text { Sommer } \\
(2013)\end{array}$ & $\begin{array}{l}\text { Erlangen } \\
\text { (Germany) }\end{array}$ & 1.5 & 25 & $2 / 23$ & $\begin{array}{l}\text { 12-67; } \\
\text { mean } 37\end{array}$ & $\begin{array}{l}\text { median: } \\
44.2 \pm \\
26.9\end{array}$ & PharmacoR & Extratemporal & $9 / 16$ & No \\
\hline $\begin{array}{l}\text { Kurwale } \\
(2015)\end{array}$ & $\begin{array}{l}\text { New Delhi } \\
\text { (India) }\end{array}$ & 1.5 & 39 & $\begin{array}{l}\text { Both (not } \\
\text { specified) }\end{array}$ & $\begin{array}{l}3-65 ; \\
\text { mean } 22\end{array}$ & $\begin{array}{l}\text { mean: } \\
14\end{array}$ & PharmacoR & Mixed & $12 / 27$ & No \\
\hline $\begin{array}{l}\text { Sommer } \\
(2015)\end{array}$ & $\begin{array}{l}\text { Erlangen } \\
\text { (Germany) }\end{array}$ & 1.5 & 69 & $\begin{array}{l}\text { Both (not } \\
\text { specified) }\end{array}$ & $28.5 \pm 15.4$ & $\begin{array}{l}\text { median: } \\
55.5 \pm \\
36.2\end{array}$ & $\begin{array}{l}51 / 69 \\
\text { PharmacoR }\end{array}$ & Mixed & $69 / 0$ & No \\
\hline $\begin{array}{l}\text { Warsi } \\
(2016)\end{array}$ & $\begin{array}{l}\text { Montreal } \\
\text { (Canada) }\end{array}$ & 3 & 39 & $39 / 0$ & $\begin{array}{l}0-18 ; \\
\text { mean } 8\end{array}$ & $>24$ & PharmacoR & Mixed & $12 / 27$ & No \\
\hline $\begin{array}{l}\text { Sacino } \\
(2016)\end{array}$ & $\begin{array}{l}\text { (Washington, } \\
\text { USA) }\end{array}$ & 1.5 & 12 & $12 / 0$ & $\begin{array}{l}0-18 \\
8.8 \pm 1.6\end{array}$ & $\begin{array}{l}\text { mean: } \\
3.5\end{array}$ & PharmacoR & Mixed & $0 / 12$ & No \\
\hline $\begin{array}{l}\text { Roessler } \\
(2016)\end{array}$ & $\begin{array}{l}\text { Erlangen } \\
\text { (Germany) }\end{array}$ & 1.5 & 415 & $\begin{array}{l}\text { Both (not } \\
\text { specified) }\end{array}$ & $\begin{array}{l}5-69 ; \\
\text { mean } 37.2\end{array}$ & $\begin{array}{l}36 ; \\
\text { range 3- } \\
10.8 \text { y }\end{array}$ & PharmacoR & Mixed & $\begin{array}{l}\text { 112/177/other } \\
\text { non specified }\end{array}$ & No \\
\hline $\begin{array}{l}\text { Sacino } \\
(2016)\end{array}$ & $\begin{array}{l}\text { (Washington, } \\
\text { USA) }\end{array}$ & 1.5 & 11 & $11 / 0$ & $9.1 \pm 2.0$ & $\begin{array}{l}5.72 ; \\
\text { range } \\
0.46- \\
10.61 \\
\text { months }\end{array}$ & PharmacoR & Mixed & $0 / 11$ & No \\
\hline $\begin{array}{l}\text { Van } \\
\text { Tonder } \\
(2018)\end{array}$ & $\begin{array}{l}\text { Liverpool } \\
\text { (UK) }\end{array}$ & & 10 & $10 / 0$ & $\begin{array}{l}7 y \\
\text { (range 2-17 } \\
\text { y). }\end{array}$ & $\begin{array}{l}\text { mean } \\
34 \\
\text { months, } \\
1-76 \\
\text { months }\end{array}$ & PharmacoR & $\begin{array}{l}\text { Extratemporal, } \\
\mathrm{HH}\end{array}$ & $10 / 0$ & No \\
\hline \multicolumn{2}{|c|}{ Total numbers } & & 867 & $\begin{array}{l}99 \\
\text { Pediatric } \\
196 \text { Adult } \\
\text { (if } \\
\text { reported) }\end{array}$ & & & & & \multicolumn{2}{|l|}{$\begin{array}{l}320 \text { Lesional } \\
413 \text { Non lesional } \\
\text { (if reported) }\end{array}$} \\
\hline
\end{tabular}


Table 2

Summary of series: final diagnosis, complete/adequate resection, surgical outcomes in terms of Engel class I

\begin{tabular}{|c|c|c|c|c|c|c|}
\hline $\begin{array}{l}\text { Author } \\
\text { (year) }\end{array}$ & Number & $\begin{array}{l}\text { Anatomopathological diagnosis } \\
\text { (lesional) }\end{array}$ & $\begin{array}{l}\text { Anatomopathological diagnosis } \\
\text { (nonlesional) }\end{array}$ & $\begin{array}{l}\text { Complete/adequate } \\
\text { resection } \\
\text { (after 1st iMRI) }\end{array}$ & $\begin{array}{l}\text { Second } \\
\text { evaluation } \\
\text { after iMRI }\end{array}$ & $\begin{array}{l}\text { Engel } \\
\text { class I }\end{array}$ \\
\hline $\begin{array}{l}\text { Buchfelder } \\
(2000)\end{array}$ & 61 & $\begin{array}{l}\text { Pilocytic astrocytoma (2), } \\
\text { Astrocytoma grade II (9), } \\
\text { gangliogliomas (4), DNET (2), } \\
\text { cavernoma (8), other (4) }\end{array}$ & $\begin{array}{l}\text { Sclerosis (9), cortical dysplasia (2), } \\
\text { gliosis (12), cortical microdysgenesis } \\
\text { (3), glioneural hamartia (2), no } \\
\text { abnormality (4) }\end{array}$ & $55 / 61(90 \%)$ & $\begin{array}{l}3 / 61 \\
(4.9 \%)\end{array}$ & $\begin{array}{l}46 / 61 \\
(75 \%)\end{array}$ \\
\hline $\begin{array}{l}\text { Buchfelder } \\
(2002)\end{array}$ & 58 & $\begin{array}{l}\text { Pilocytic astrocytoma (6), } \\
\text { astrocytoma grade II (4), } \\
\text { gangliogliomas (2), DNET (2), } \\
\text { cavernoma (7), other (8) }\end{array}$ & $\begin{array}{l}\text { Sclerosis (10), dysplasia (3), gliosis (7), } \\
\text { other (9) }\end{array}$ & $53 / 58(91.3 \%)$ & $\begin{array}{l}3 / 58 \\
(5.2 \%)\end{array}$ & $\begin{array}{l}47 / 58 \\
(81 \%)\end{array}$ \\
\hline $\begin{array}{l}\text { Kaibara } \\
(2002)\end{array}$ & 14 & NA & $\begin{array}{l}\text { Sclerosis (4), heterotopia (1), } \\
\text { other/normal (9) }\end{array}$ & $7 / 14(50 \%)$ & $\begin{array}{l}7 / 14 \\
(50 \%)\end{array}$ & $\begin{array}{l}13 / 14 \\
(92.8 \%)\end{array}$ \\
\hline $\begin{array}{l}\text { Schwarz } \\
(2002)\end{array}$ & 5 & DNET (1) & Sclerosis (3), dysplasia (1) & $0 / 5(0 \%)$ & $\begin{array}{l}5 / 5 \\
(100 \%)\end{array}$ & $\begin{array}{l}5 / 5 \\
(100 \%)\end{array}$ \\
\hline $\begin{array}{l}\text { Walker } \\
(2002)\end{array}$ & 13 & $\begin{array}{l}\text { Gangliogliomas (5), DNET (3), HH } \\
\text { (1) }\end{array}$ & Cortical dysplasia (4) & $8 / 13(61.5 \%)$ & $\begin{array}{l}5 / 13 \\
(39.5 \%)\end{array}$ & $\begin{array}{l}4 / 13 \\
(30.7 \%)\end{array}$ \\
\hline \multirow{2}{*}{$\begin{array}{l}\text { Kelly } \\
(2005)\end{array}$} & \multirow[t]{2}{*}{70} & $51(73 \%)$ TLE & \multirow[t]{2}{*}{ Sclerosis (39), dysplasia (9), other (10) } & \multirow[t]{2}{*}{$51 / 70(72.8 \%)$} & \multirow{2}{*}{$\begin{array}{l}18 / 70 \\
(25.7 \%)\end{array}$} & \multirow{2}{*}{$\begin{array}{l}41 / 70 \\
(58.5 \%)\end{array}$} \\
\hline & & $\begin{array}{l}\text { Gangliogliomas (4), DNET (2), } \\
\text { other (3) }\end{array}$ & & & & \\
\hline $\begin{array}{l}\text { Sun } \\
(2011)\end{array}$ & 36 & Cavernoma (36) & Cavernoma & $36 / 36(100 \%)$ & $0 \%$ & $\begin{array}{l}17 / 20 \\
(85 \%)\end{array}$ \\
\hline $\begin{array}{l}\text { Sommer } \\
(2013)\end{array}$ & 25 & $\begin{array}{l}\text { Gangliogliomas (2), DNET (2), } \\
\text { Cavernoma (5) }\end{array}$ & Dysplasia (7), gliosis (5), other (4) & $20 / 25(80 \%)$ & $\begin{array}{l}5 / 25 \\
(20 \%)\end{array}$ & $\begin{array}{l}19 / 25 \\
(76 \%)\end{array}$ \\
\hline $\begin{array}{l}\text { Kurwale } \\
(2015)\end{array}$ & 39 & $\begin{array}{l}\text { Pilocytic astrocytoma (2), DNET } \\
\text { (4), hamartoma (3); other (3) }\end{array}$ & Sclerosis (7), dysplasia (11), gliosis (9) & $30 / 39(77 \%)$ & $\begin{array}{l}5 / 39 \\
(12.8 \%)\end{array}$ & $\begin{array}{l}33 / 39 \\
(84.6 \%)\end{array}$ \\
\hline $\begin{array}{l}\text { Sommer } \\
(2015)\end{array}$ & 69 & Gangliogliomas (69) & N/A & $33 / 48(68.7 \%)$ & $\begin{array}{l}9 / 48 \\
(18.7 \%)\end{array}$ & $\begin{array}{l}42 / 60 \\
(70 \%)\end{array}$ \\
\hline $\begin{array}{l}\text { Warsi } \\
(2016)\end{array}$ & 39 & $\begin{array}{l}\text { Tumor (no details, 11), cavernoma } \\
\text { (1) }\end{array}$ & $\begin{array}{l}\text { Sclerosis (3), dysplasia (12), ischemia } \\
\text { (8), other (4) }\end{array}$ & $32 / 39(82 \%)$ & $\begin{array}{l}7 / 39 \\
(21.8 \%)\end{array}$ & $\begin{array}{l}34 / 39 \\
\text { (87.1\% } \\
\text { Engel I } \\
\text { and II } \\
\text { included } \\
\text { together) }\end{array}$ \\
\hline $\begin{array}{l}\text { Sacino } \\
(2016)\end{array}$ & 12 & NA & Dysplasia (12) & $7 / 12(58.3 \%)$ & $\begin{array}{l}5 / 12 \\
(41.6 \%)\end{array}$ & $\begin{array}{l}11 / 12 \\
(91.6 \%)\end{array}$ \\
\hline $\begin{array}{l}\text { Roessler } \\
(2016)\end{array}$ & 415 & $\begin{array}{l}\text { LEAT (GG, DNET- 67), cavernoma } \\
\text { (45) }\end{array}$ & Sclerosis (146), dysplasia (31) & $369 / 415(89 \%)$ & $\begin{array}{l}46 / 415 \\
(11 \%)\end{array}$ & $\begin{array}{l}272 / 374 \\
(72 \%) \\
\text { with } \\
\text { complete } \\
\text { follow- } \\
\text { up }\end{array}$ \\
\hline $\begin{array}{l}\text { Sacino } \\
(2016)\end{array}$ & 11 & NA & Dysplasia (11) & $7 / 11(63.6 \%)$ & $\begin{array}{l}4 / 11 \\
(36.3 \%)\end{array}$ & $\begin{array}{l}9 / 11 \\
(82 \%)\end{array}$ \\
\hline $\begin{array}{l}\text { Van } \\
\text { Tonder } \\
\text { (2018) }\end{array}$ & 10 & Hypothalamic hamartoma & Hypothalamic hamartoma (10) & $6 / 10(60 \%)$ & $\begin{array}{l}4 / 10 \\
(40 \%)\end{array}$ & $\begin{array}{l}8 / 10 \\
(80 \%)\end{array}$ \\
\hline \multicolumn{7}{|c|}{$\begin{array}{l}\text { Lesional: Pilocytic astrocytoma }=10 \text {, astrocytoma grade } \|=13, \mathrm{DNET}=16, \text { Gangliogliomas }=84, \mathrm{DNET}+\mathrm{Gangliogliomas}=167, \mathrm{HH}=14, \text { Cavernoma }=102 \\
\text { and Other= } 18\end{array}$} \\
\hline
\end{tabular}

The data extraction for NLE only is presented in the supplementary materiel.

\section{Terminology issues}

In order to respect definitions reported by authors of included studies, two groups were analyzed: LE and NLE.

Lesional epilepsy was constantly defined as related to easily detected and well-defined pathologies on MRI: tumors (i.e. pilocytic astrocytoma, astrocytoma, DNET, gangliogliomas, DNET+gangliogliomas), cavernous malformations etc.

Intractable epilepsy is however frequently related to other types of pathology, where the epileptic focus and epileptogenic zone can become subtler or poorly defined on cerebral MRI. Such pathologies include sclerosis, dysplasia, gliosis, cortical microdysgenesis, glioneural hamartia, ischemia etc. This group has 
consistently been reported as NLE. Of note, no visible abnormality on preoperative MRI was included in this group.

\section{Statistical analysis}

Weighted summary rates were determined using meta-analysis models. The test for heterogeneity was performed for each meta-analysis. In cases of heterogeneity, a binary random-effects model (DerSimonian-Laird method) was used in some of the analysis assuming that the included studies were a random sample from hypothetical population; otherwise, a binary fixed-effects model with inverse variance weighting was employed.

We used OpenMeta (Analyst) from the Agency for Healthcare Research and Quality to perform our analyses. Pooled estimates using meta-analytical techniques were obtained for the outcome of complete/adequate resection before and after using iMRI, as well as for Engel class I outcome (when reported separately), which followed binomial distributions.

\section{Results}

\section{Populations}

The mean follow-up periods as well as the basic demographic data are reported in Table 1.

Of note, pediatric cases were exclusively reported by four studies $(23,28,30,31)$, comprising 72 cases. All the others described both adult and pediatric cases in different proportions.

The results for NLE only are presented in the supplementary materiel.

\section{Surgical indications}

Overall number of cases reported for LE was 320 and for NLE was 413 patients(1, 18-31) (cases where individual clear anatomopathological diagnosis was specified).

Most common surgical indication in LE (figure 2, upper part) was dysembrioplastic neuroepithelial tumor (DNET) and gangliogliomas ( $\mathrm{n}=167)$, cavernomas $(n=102)$, gangliogliomas $(n=84)$, DNET $(n=16), H H(n=14)$, WHO grade II astrocytoma $(n=13)$ and pilocytic astrocytoma $(n=10)(1,18-31)$. In NLE this frequently included (figure 2, lower part) sclerosis $(n=221)$, dysplasia $(n=103)$, ischemia $(n=8)$ and gliosis $(n=33)(1,18-31)$.

Pediatric studies reported mainly surgery for tumor, cavernomas and hypothalamic hamartomas $(\mathrm{HH})$ or dysplasia, ischemia, sclerosis and other causes(23, $28,30,31)$.

Complete or adequate resection based on initial preoperative neuroimaging assessment

Pooled overall initial complete or adequate resection rate was $74.8 \%$ (95\% confidence interval (Cl) 67.3-82.3\%). A binary random-effects model was used ( $\mathrm{c}<$ $\left.0.001, I^{\wedge} 2=88.84\right)$.

\section{Further intervention after iMRI}

Hundred and twenty-six cases out of 820 underwent additional resection after iMRI, for an overall rate of 24.4\% (range 16.9-31.8). A binary random-effects model was used $\left(p<0.001, \iota^{\wedge} 2=85.32\right.$, figure 3 , Table 3$)$. 
Table 3

Summary of series: complications, need for reintervention, main message

\begin{tabular}{|c|c|c|c|c|c|}
\hline $\begin{array}{l}\text { Author } \\
\text { (year) }\end{array}$ & Number & Complications & Engel class I: & $\begin{array}{l}\text { Surgical indications } \\
\text { for reintervention }\end{array}$ & Main results and/or message \\
\hline \multirow[t]{2}{*}{$\begin{array}{l}\text { Buchfelder } \\
(2000)\end{array}$} & \multirow[t]{2}{*}{61} & \multirow[t]{2}{*}{-} & \multirow[t]{2}{*}{$38 / 61(62.3 \%)$} & \multirow[t]{2}{*}{$3 / 61(4.9 \%)$} & $\begin{array}{l}\text { A second look }(n=3) \text { could increase the rate of total resection in } \\
\text { the lesional cases from } 79-90 \% \text {. }\end{array}$ \\
\hline & & & & & $\begin{array}{l}\text { iMRI allowed evaluating the extent of resection and/or } \\
\text { disconnection. }\end{array}$ \\
\hline \multirow[t]{2}{*}{$\begin{array}{l}\text { Buchfelder } \\
(2002)\end{array}$} & \multirow[t]{2}{*}{58} & $\begin{array}{l}\text { LE: } 4 / 29 \\
(13.8 \%)\end{array}$ & $\begin{array}{l}\text { LE: } n=22 / 29 \\
(75.9 \%)\end{array}$ & \multirow{2}{*}{$\begin{array}{l}\text { LE: } n=2 \\
\text { NLE: } n=1\end{array}$} & $\begin{array}{l}\text { Gliomas total resection rate: } 73 \% \text {-> } 87 \% \text {; extension in eloquent } \\
\text { areas precluded TR }\end{array}$ \\
\hline & & $\begin{array}{l}\text { NLE: } 9 / 29 \\
(31 \%)\end{array}$ & $\begin{array}{l}\text { NLE: } n=17 / 29 \\
(58.6 \%)\end{array}$ & & Overall: $16 \%$ postoperative changes in resection volume \\
\hline $\begin{array}{l}\text { Kaibara } \\
(2002)\end{array}$ & 14 & $1 / 14(7.1 \%)$ & $13 / 14(93 \%)$ & $7 / 14(50 \%)$ & $\begin{array}{l}\text { In TLE, iMRI can identify residual mesial temporal lobe targets } \\
\text { before craniotomy closure. }\end{array}$ \\
\hline $\begin{array}{l}\text { Schwarz } \\
(2002)\end{array}$ & 5 & $0 / 5(0 \%)$ & $5 / 5(100 \%)$ & - & $\begin{array}{l}\text { iMRI is an useful adjunct for surgical treatment of MTLE, and } \\
\text { perhaps the most reliable method of standardizing a complete } \\
\text { hippocampectomy. }\end{array}$ \\
\hline $\begin{array}{l}\text { Walker } \\
(2002)\end{array}$ & 13 & $2 / 13(15.4 \%)$ & $5 / 13(38.5 \%)$ & $5 / 13(38.5 \%)$ & $\begin{array}{l}\text { iMRI is a safe and effective adjunct for the surgical treatment of } \\
\text { benign intracranial lesions presenting with seizures. }\end{array}$ \\
\hline \multirow[t]{2}{*}{$\begin{array}{l}\text { Kelly } \\
(2005)\end{array}$} & \multirow[t]{2}{*}{70} & \multirow[t]{2}{*}{$1 / 70(1.4 \%)$} & $\begin{array}{l}\text { LE: } \mathrm{n}=7 / 7 \\
(100 \%)\end{array}$ & \multirow{2}{*}{$\begin{array}{l}\text { LE: } n=3 \\
\text { NLE: } n=15\end{array}$} & \multirow{2}{*}{$\begin{array}{l}\text { Benefit in patients with cortical dysplasia. } \\
\text { Decrease likehood of distant reintervention in TLE. }\end{array}$} \\
\hline & & & $\begin{array}{l}\text { NLE: } n=34 / 59 \\
(57.6 \%)\end{array}$ & & \\
\hline \multirow{2}{*}{$\begin{array}{l}\text { Sun } \\
(2011)\end{array}$} & \multirow[t]{2}{*}{36} & \multirow[t]{2}{*}{$0 \%$} & \multirow[t]{2}{*}{ LE: $7 / 9(78 \%)$} & \multirow{2}{*}{$\begin{array}{l}2 \text { patients with } \\
\text { further resection in } \\
\text { extratemporal } \\
\text { location }\end{array}$} & Supratentorial cavernomas in eloquent brain areas. \\
\hline & & & & & $\begin{array}{l}\text { Sparing parts of the surrounding hypointense rim in } 10 \text { patients to } \\
\text { prevent deficits. }\end{array}$ \\
\hline \multirow{2}{*}{$\begin{array}{l}\text { Sommer } \\
(2013)\end{array}$} & \multirow[t]{2}{*}{25} & Transient 20\% & $72 \%$ & \multirow[t]{2}{*}{$20 \%$} & \multirow{2}{*}{$\begin{array}{l}\text { Benefit in patients with extratemporal epilepsies close to eloquent } \\
\text { areas (distance less than } 20 \mathrm{~mm} \text { ). Improved clinical outcomes. } \\
\text { Could potentially avoid awake surgery. }\end{array}$} \\
\hline & & $\begin{array}{l}\text { Permanent } \\
12 \%\end{array}$ & (all lesional) & & \\
\hline \multirow[t]{2}{*}{$\begin{array}{l}\text { Kurwale } \\
(2015)\end{array}$} & \multirow[t]{2}{*}{39} & \multirow[t]{2}{*}{ 4/39 (10.3) } & \multirow[t]{2}{*}{$77 \%$} & \multirow{2}{*}{$\begin{array}{l}4 \text { lesions on eloquent } \\
\text { area or less than } 1 \\
\mathrm{~mm} \text { apart }\end{array}$} & $\begin{array}{l}\text { In LE, iMRI modified operative strategy resulting in increase } \\
\text { resection in } 5 / 23(21 \%) \text {. }\end{array}$ \\
\hline & & & & & In LE, iMRI increased the extent of resection. \\
\hline \multirow[t]{2}{*}{$\begin{array}{l}\text { Sommer } \\
(2015)\end{array}$} & \multirow[t]{2}{*}{69} & $\begin{array}{l}\text { Transient } 1 \\
(1.4 \%)\end{array}$ & \multirow[t]{2}{*}{$42 / 60(72 \%)$} & $\begin{array}{l}\text { Remnant tumor in } \\
28 \%\end{array}$ & $\begin{array}{l}\text { In GG surgery, the authors raised the rate of complete resection by } \\
19 \% \text {. }\end{array}$ \\
\hline & & $\begin{array}{l}\text { Permanent } 4 \\
(5.8 \%)\end{array}$ & & & Remnant tumor identified using iMRI in 13/46 (28\%). \\
\hline $\begin{array}{l}\text { Warsi } \\
(2016)\end{array}$ & 39 & $2 / 39(5.1 \%)$ & $\begin{array}{l}34 / 39(87.2 \%) \\
\text { Engel I and II } \\
\text { reported }\end{array}$ & - & $\begin{array}{l}\text { Retrospective analysis with and w-iMRI: Engel I/II outcome more } \\
\text { frequent in iMRI. }\end{array}$ \\
\hline & & & together & & At 2-years similar results. Does not favor iMRI in pediatric epilepsy. \\
\hline $\begin{array}{l}\text { Sacino } \\
(2016)\end{array}$ & 12 & - & $11 / 12(92 \%)$ & $5 / 12(42 \%)$ & $\begin{array}{l}\text { iMRI precluded the need for repeat surgery in pediatric patients } \\
\text { with focal cortical dysplasia. }\end{array}$ \\
\hline $\begin{array}{l}\text { Roessler } \\
(2016)\end{array}$ & 415 & $16.3 \%$ & $72.7 \%$ & $49 / 415(1.8 \%)$ & $\begin{array}{l}\text { Increased likelihood of Engel I found in cavernoma, followed by } \\
\text { hippocampal sclerosis and long-term epilepsy-associated tumor. }\end{array}$ \\
\hline & & & & & $\begin{array}{l}\text { Higher resection volume associated with a higher chance of } \\
\text { favorable seizure outcome, especially in long-term epilepsy- } \\
\text { associated tumor or diffuse gliomas. }\end{array}$ \\
\hline $\begin{array}{l}\text { Sacino } \\
(2016)\end{array}$ & 11 & $4 / 11(36 \%)$ & 9/11 (82\%) & $4 / 11(36 \%)$ & $\begin{array}{l}\text { For resection of lesions in peri-eloquent cortex, the incorporation } \\
\text { of iMRI led to elevated rates of gross total resection and } \\
\text { postoperative seizure freedom. }\end{array}$ \\
\hline $\begin{array}{l}\text { Van } \\
\text { Tonder }\end{array}$ & 10 & $0 / 10(0 \%)$ & $8 / 10(80 \%)$ & $4 / 10(40 \%)$ & $\begin{array}{l}\text { In } \mathrm{HH} \text {, iMRI can be used to tailor the ideal tumor resection based } \\
\text { on the anatomy of the lesion. }\end{array}$ \\
\hline (2018) & & & & & \\
\hline
\end{tabular}

\section{Complete or adequate resection based on iMRI with further intervention}

Pooled overall complete or adequate resection rate after iMRI use was $97.6 \%$ (95\% confidence interval (Cl) 95.8-99.3\%). A binary random-effects model was used $\left(p=0.003, I^{\wedge} 2=56.852\right.$, figure 3 , Table 3$)$.

Clinical outcome in terms of Engel class I at last follow-up 
Pooled overall Engel class I at last follow-up was 77.1\% (95\% confidence interval $(\mathrm{Cl}) 71-83.2 \%)$. A binary random-effects model was used $(\mathrm{p}<0.001$, I^2= 68.134, figure 4).

\section{Discussion}

In the present meta-analysis, we report 15 studies, encompassing 867 patients, with various types of LE and NLE. Most common surgical indications in LE were dysembrioplastic neuroepithelial tumor (DNET) and gangliogliomas included together or separately, cavernomas, $\mathrm{HH}$, or primary brain tumors and in NLE were sclerosis, dysplasia, ischemia and gliosis. With the use of iMRI, the rate of complete or adequate microsurgical resection significantly increased from $74.8 \%(67.3-82.3 \%)$ to $97.6 \%$ (95.8-99.3\%). This was translated in an overall clinical benefit of Engel class I at last follow-up of $77.1 \%$ (71-83.2\%). While this meta-analysis included various types of epilepsy, the favorable clinical outcome is fairly comparable to what one would expect in temporal lobe epilepsy (TLE), which is universally considered to have the best postoperative seizure control.

The surgical aim of resective epilepsy surgery is either complete excision or disconnection of the epileptic network, with preservation of eloquent cortex(32). In order to achieve this, presurgical correct diagnosis is mandatory. Modern epileptologists can use multimodal diagnostic tools, including semiology analysis, electrophysiological recordings, functional testing and complex neuroimaging techniques(32). All these remain complementary and should define the location and boundaries of the epileptic zone. In challenging cases, stereo-electroencephalography (SEEG) by depth electrodes is considered of major help for evaluation and tailored navigated resection, even in patients with non-lesional or extratemporal focal epilepsy(33). In fact, it has been already acknowledged that presence of a lesion does not necessarily correlate with an epileptogenic network solely driven by that particular lesion. Moreover, a lesion itself can recruit and further drive other epileptogenic zones in the brain, making diagnosis even more challenging(34, 35). The clinical aim of resective epilepsy surgery remains the best possible seizure control, while experiencing the least possible neurologic or neuropsychological impairment. This is achieved by a maximized resection, which is mandatory for good seizure outcome.

Surgically accessible pathologies associated with medically refractory epilepsy often include hippocampal sclerosis, long-term epilepsy-associated tumors, cavernous malformations or focal cortical dysplasia(36, 37). Presence of a visible lesion on MRI correlates with better postsurgical outcomes, as does complete surgical excision of such lesions $(38,39)$. In contrast, intrinsic epilepsy-associated tumors might have diffuse borders and less accessible anatomical locations, such as adjacent to motor or speech areas $(22,25)$.

Optimal resection goals are mostly attained in temporal locations, translating into good surgical outcomes as opposed with those in extratemporal ones(27). In a recent meta-analysis(40) on long-term postsurgical outcomes seizure freedom was reported in $64 \%$ of TLE and only $34 \%$ in extra TLE, particularly for cortical malformations. Of note, one of the important reasons was incomplete removal, due to proximity to eloquent motor areas and white matter tracts. In this context, iMRI can be a valuable therapeutic adjunct, together with neuronavigation. Its utility should be seen in the context of the specific pathology related to medically intractable seizures. In TLE, iMRI can document the extent of mesial and neocortical resections as alternative to classical tailored anterior or posterior temporal lobe resections rather than standard lobectomy in attempt to maximize preservation of adjacent parenchyma(18).

In extra TLE, a variety of histopathological findings can be identified. Not all of them are necessarily well defined on neuroimaging (in contrast to hippocampal sclerosis for example). Some are further located near functional cortex or fiber tracts and thus are not necessarily amenable to complete resection without neurological impairment(25). Combined with BOLD and tractography, iMRI can offer a higher extent of resection. It can further compensate the brain shift and can adjust inaccuracy of coregistration, improve functional mapping and fiber tracking.

In cortical dysplasia, the concept of seizure outcome as related to extent of resection is also applicable. iMRI can be a valuable tool, as it is often more subtle and difficult to visually differentiate cortical dysplasia from normal brain parenchyma, in terms of intraoperative macroscopic aspects(1), causing even in the experienced neurosurgeon a feeling of false complete resections(21).

\section{Limitations}

One of the limitations is the retrospective nature of the included studies, the small sample size of each of them, as well as the lack of randomized controlled trials. A second limitation is the use of multiple surgical procedures, depending on the main pathology, inside the same report. A third limitation is that not all studies included the complete spectrum of pathologies. A fourth limitation is that we cannot comment on the surgeon's personal insight, leading to the decision-making. A fifth limitation is related to a potential selection bias of choosing the microsurgical approach, while including iMRI in the surgical armamentarium. Moreover, there was a progressive increase in the iMRI field during time; as such, our results have to be balanced with the constant evolution of intraoperative technologies. Only one study included in the present meta-analysis used 3 T iMRI. An open question is what should be the minimum postoperative follow-up in these cases (e.g. 12 months?). A technical limitation can be related to errors during calculation of functional data, target registration of the navigation systems, registrations between pre- and postoperative MRI etc. As an alternative for iMRI and neuronavigation, in selected cases, awake craniotomy represents a valuable option in experienced centers(47). A further limitation relates to specific terminology of what some authors consider as LE, while other as NLE. A last, yet important limitation, is the lack of randomized controlled trials, which would increase the level of evidence.

\section{Conclusions}

Intraoperative MRI provides an update in terms of neuroimaging during general anesthesia with the patient positioned for surgery. Combined with functional neuronavigation, it helps in evaluating the extent of resection, change or adapt the surgical plan, while correcting for brain shift, and potentially evaluates the presence of intraoperative complications. Initial extent of resection can sometimes be overestimated. In this sense, the present review suggests higher complete/adequate resection rates after performing an iMRI. When taking advantage of this recent technological innovation, the rate of complete or adequate resection might increased from an overall rate of 74.8-97.6\%. This could potentially translate in an overall clinical benefit of Engel class I at last follow-up of 
77.1\%. In the largest reported series of the present meta-analysis, increased likelihood of Engel I was found in cavernoma, followed by hippocampal sclerosis and long-term epilepsy-associated tumor. However, the reader should take into account that such data are not provided by randomized controlled trials and that such conclusions should be carefully interpreted.

Incorporating surgical robots will increase surgical precision and accuracy, allowing precise removal and potentially further modulation of abnormal tissue. Randomized controlled trials are necessary to evaluate the real impact of such new technologies.

\section{Declarations Conflict of interest:}

none

\section{Acknowledgments:}

Constantin Tuleasca gratefully acknowledges the receipt of a grant "Jeune Chercheur en Recherche Clinique" from the University of Lausanne (Unil) and Lausanne University Hospital (CHUV).

\section{Financial support:}

Lausanne University Hospital (CHUV) and University of Lausanne (Unil) for CT. JZ undertook this at UCL/UCLHT who received a proportion of funding from the Department of Health's NIHR Biomedical Research Centre funding scheme.

\section{Disclosures:}

none as related to the present manuscript and topic

\section{References}

1. Kelly JJ, Hader WJ, Myles ST, Sutherland GR. Epilepsy surgery with intraoperative MRI at 1.5 T. Neurosurgery clinics of North America. 2005 Jan;16(1):173-83. PubMed PMID: 15561537.

2. Fisher RS, Acevedo C, Arzimanoglou A, Bogacz A, Cross JH, Elger CE, et al. ILAE official report: a practical clinical definition of epilepsy. Epilepsia. 2014 Apr;55(4):475-82. PubMed PMID: 24730690.

3. Fiest KM, Sauro KM, Wiebe S, Patten SB, Kwon CS, Dykeman J, et al. Prevalence and incidence of epilepsy: A systematic review and meta-analysis of international studies. Neurology. 2017 Jan 17;88(3):296-303. PubMed PMID: 27986877. Pubmed Central PMCID: 5272794.

4. Kwan P, Sander JW. The natural history of epilepsy: an epidemiological view. Journal of neurology, neurosurgery, and psychiatry. 2004 Oct;75(10):137681. PubMed PMID: 15377680. Pubmed Central PMCID: 1738749.

5. Spencer DD, Spencer SS. Surgery for epilepsy. Neurologic clinics. 1985 May;3(2):313-30. PubMed PMID: 3927130.

6. Bengzon AR, Rasmussen T, Gloor P, Dussault J, Stephens M. Prognostic factors in the surgical treatment of temporal lobe epileptics. Neurology. 1968 Aug;18(8):717-31. PubMed PMID: 5692332.

7. Clusmann H, Kral T, Fackeldey E, Blumcke I, Helmstaedter C, von Oertzen J, et al. Lesional mesial temporal lobe epilepsy and limited resections: prognostic factors and outcome. Journal of neurology, neurosurgery, and psychiatry. 2004 Nov;75(11):1589-96. PubMed PMID: 15489392. Pubmed Central PMCID: 1738802.

8. Clusmann H, Schramm J, Kral T, Helmstaedter C, Ostertun B, Fimmers R, et al. Prognostic factors and outcome after different types of resection for temporal lobe epilepsy. Journal of neurosurgery. 2002 Nov;97(5):1131-41. PubMed PMID: 12450036.

9. Schramm J, Kral T, Grunwald T, Blumcke I. Surgical treatment for neocortical temporal lobe epilepsy: clinical and surgical aspects and seizure outcome. Journal of neurosurgery. 2001 Jan;94(1):33-42. PubMed PMID: 11147895.

10. Spencer SS. When should temporal-lobe epilepsy be treated surgically? The Lancet Neurology. 2002 Oct;1(6):375-82. PubMed PMID: 12849399.

11. Zentner J, Hufnagel A, Wolf HK, Ostertun B, Behrens E, Campos MG, et al. Surgical treatment of temporal lobe epilepsy: clinical, radiological, and histopathological findings in 178 patients. Journal of neurology, neurosurgery, and psychiatry. 1995 Jun;58(6):666-73. PubMed PMID: 7608662. Pubmed Central PMCID: 1073541.

12. Engel J, Jr. Classification of epileptic disorders. Epilepsia. 2001 Mar;42(3):316. PubMed PMID: 11442146.

13. Wyler AR, Hermann BP, Somes G. Extent of medial temporal resection on outcome from anterior temporal lobectomy: a randomized prospective study. Neurosurgery. 1995 Nov;37(5):982-90; discussion 90-1. PubMed PMID: 8559349.

14. Berkovic SF. Surgical treatment of temporal lobe epilepsy. Journal of neurology, neurosurgery, and psychiatry. 2002 Nov;73(5):470. PubMed PMID: 12397134. Pubmed Central PMCID: 1738099.

15. Englot DJ, Han SJ, Berger MS, Barbaro NM, Chang EF. Extent of surgical resection predicts seizure freedom in low-grade temporal lobe brain tumors. Neurosurgery. 2012 Apr;70(4):921-8; discussion 8. PubMed PMID: 21997540. 
16. Giulioni M, Rubboli G, Marucci G, Martinoni M, Volpi L, Michelucci R, et al. Seizure outcome of epilepsy surgery in focal epilepsies associated with temporomesial glioneuronal tumors: lesionectomy compared with tailored resection. Journal of neurosurgery. 2009 Dec;111(6):1275-82. PubMed PMID: 19408976.

17. Mclnerney J, Roberts DW. Frameless stereotaxy of the brain. The Mount Sinai journal of medicine, New York. 2000 Sep;67(4):300-10. PubMed PMID: 11021780 .

18. Buchfelder M, Fahlbusch R, Ganslandt O, Stefan H, Nimsky C. Use of intraoperative magnetic resonance imaging in tailored temporal lobe surgeries for epilepsy. Epilepsia. 2002 Aug;43(8):864-73. PubMed PMID: 12181005.

19. Buchfelder M, Ganslandt O, Fahlbusch R, Nimsky C. Intraoperative magnetic resonance imaging in epilepsy surgery. Journal of magnetic resonance imaging: JMRI. 2000 Oct;12(4):547-55. PubMed PMID: 11042635.

20. Kaibara T, Myles ST, Lee MA, Sutherland GR. Optimizing epilepsy surgery with intraoperative MR imaging. Epilepsia. 2002 Apr;43(4):425-9. PubMed PMID: 11952774.

21. Kurwale NS, Chandra SP, Chouksey P, Arora A, Garg A, Sarkar C, et al. Impact of intraoperative MRI on outcomes in epilepsy surgery: preliminary experience of two years. British journal of neurosurgery. 2015 Jun;29(3):380-5. PubMed PMID: 25659959.

22. Roessler K, Sommer B, Grummich P, Coras R, Kasper BS, Hamer HM, et al. Improved resection in lesional temporal lobe epilepsy surgery using neuronavigation and intraoperative MR imaging: favourable long term surgical and seizure outcome in 88 consecutive cases. Seizure. 2014 Mar;23(3):201-7. PubMed PMID: 24355699.

23. Sacino MF, Ho CY, Murnick J, Keating RF, Gaillard WD, Oluigbo CO. The role of intraoperative MRI in resective epilepsy surgery for peri-eloquent cortex cortical dysplasias and heterotopias in pediatric patients. Neurosurgical focus. 2016 Mar;40(3):E16. PubMed PMID: 26926056.

24. Schwartz TH, Marks D, Pak J, Hill J, Mandelbaum DE, Holodny Al, et al. Standardization of amygdalohippocampectomy with intraoperative magnetic resonance imaging: preliminary experience. Epilepsia. 2002 Apr;43(4):430-6. PubMed PMID: 11952775.

25. Sommer B, Kasper BS, Coras R, Blumcke I, Hamer HM, Buchfelder M, et al. Surgical management of epilepsy due to cerebral cavernomas using neuronavigation and intraoperative MR imaging. Neurological research. 2013 Dec;35(10):1076-83. PubMed PMID: 24083819. Pubmed Central PMCID: 3823933.

26. Sommer B, Roessler K, Rampp S, Hamer HM, Blumcke I, Stefan H, et al. Magnetoencephalography-guided surgery in frontal lobe epilepsy using neuronavigation and intraoperative MR imaging. Epilepsy research. 2016 Oct;126:26-36. PubMed PMID: 27423016.

27. Sun GC, Chen XL, Zhao Y, Wang F, Song ZJ, Wang YB, et al. Intraoperative MRI with integrated functional neuronavigation-guided resection of supratentorial cavernous malformations in eloquent brain areas. Journal of clinical neuroscience: official journal of the Neurosurgical Society of Australasia. 2011 Oct;18(10):1350-4. PubMed PMID: 21719294.

28. van Tonder L, Burn S, lyer A, Blair J, Didi M, Carter M, et al. Open resection of hypothalamic hamartomas for intractable epilepsy revisited, using intraoperative MRI. Child's nervous system: ChNS : official journal of the International Society for Pediatric Neurosurgery. 2018 Sep;34(9):1663-73. PubMed PMID: 29752488.

29. Walker DG, Talos F, Bromfield EB, Black PM. Intraoperative magnetic resonance for the surgical treatment of lesions producing seizures. Journal of clinical neuroscience: official journal of the Neurosurgical Society of Australasia. 2002 Sep;9(5):515-20. PubMed PMID: 12383406.

30. Warsi NM, Lasry O, Farah A, Saint-Martin C, Montes JL, Atkinson J, et al. 3-T intraoperative MRI (iMRI) for pediatric epilepsy surgery. Child's nervous system: ChNS : official journal of the International Society for Pediatric Neurosurgery. 2016 Dec;32(12):2415-22. PubMed PMID: 27757571.

31. Sacino MF, Ho CY, Murnick J, Tsuchida T, Magge SN, Keating RF, et al. Intraoperative MRI-guided resection of focal cortical dysplasia in pediatric patients: technique and outcomes. Journal of neurosurgery Pediatrics. 2016 Jun;17(6):672-8. PubMed PMID: 26919314.

32. Rosenow F, Luders H. Presurgical evaluation of epilepsy. Brain: a journal of neurology. 2001 Sep;124(Pt 9):1683-700. PubMed PMID: 11522572.

33. Thorsteinsdottir J, Vollmar C, Tonn JC, Kreth FW, Noachtar S, Peraud A. Outcome after individualized stereoelectroencephalography (sEEG) implantation and navigated resection in patients with lesional and non-lesional focal epilepsy. Journal of neurology. 2019 Apr;266(4):910-20. PubMed PMID: 30701313.

34. Luders HO, Najm I, Nair D, Widdess-Walsh P, Bingman W. The epileptogenic zone: general principles. Epileptic disorders: international epilepsy journal with videotape. 2006 Aug;8 Suppl 2:S1-9. PubMed PMID: 17012067.

35. Abel TJ, Woodroffe RW, Nourski KV, Moritani T, Capizzano AA, Kirby P, et al. Role of the temporal pole in temporal lobe epilepsy seizure networks: an intracranial electrode investigation. Journal of neurosurgery. 2018 Jul;129(1):165-73. PubMed PMID: 29027858.

36. Englot DJ, Berger MS, Barbaro NM, Chang EF. Factors associated with seizure freedom in the surgical resection of glioneuronal tumors. Epilepsia. 2012 Jan;53(1):51-7. PubMed PMID: 21933181.

37. Jobst BC, Cascino GD. Resective epilepsy surgery for drug-resistant focal epilepsy: a review. Jama. 2015 Jan 20;313(3):285-93. PubMed PMID: 25602999.

38. Chandra SP, Bal CS, Jain S, Joshua SP, Gaikwad S, Garg A, et al. Intraoperative coregistration of magnetic resonance imaging, positron emission tomography, and electrocorticographic data for neocortical lesional epilepsies may improve the localization of the epileptogenic focus: a pilot study. World neurosurgery. 2014 Jul-Aug;82(1-2):110-7. PubMed PMID: 23438971.

39. Palmini A, Andermann F, Olivier A, Tampieri D, Robitaille Y. Focal neuronal migration disorders and intractable partial epilepsy: results of surgical treatment. Annals of neurology. 1991 Dec;30(6):750-7. PubMed PMID: 1789692.

40. Tellez-Zenteno JF, Dhar R, Wiebe S. Long-term seizure outcomes following epilepsy surgery: a systematic review and meta-analysis. Brain: a journal of neurology. 2005 May;128(Pt 5):1188-98. PubMed PMID: 15758038. 
41. van Breemen MS, Wilms EB, Vecht CJ. Epilepsy in patients with brain tumours: epidemiology, mechanisms, and management. The Lancet Neurology. 2007 May;6(5):421-30. PubMed PMID: 17434097.

42. Luyken C, Blumcke I, Fimmers R, Urbach H, Wiestler OD, Schramm J. Supratentorial gangliogliomas: histopathologic grading and tumor recurrence in 184 patients with a median follow-up of 8 years. Cancer. 2004 Jul 1;101(1):146-55. PubMed PMID: 15222000.

43. Majores M, von Lehe M, Fassunke J, Schramm J, Becker AJ, Simon M. Tumor recurrence and malignant progression of gangliogliomas. Cancer. 2008 Dec 15;113(12):3355-63. PubMed PMID: 18988291.

44. Sommer B, Wimmer C, Coras R, Blumcke I, Lorber B, Hamer HM, et al. Resection of cerebral gangliogliomas causing drug-resistant epilepsy: short- and long-term outcomes using intraoperative MRI and neuronavigation. Neurosurgical focus. 2015 Jan;38(1):E5. PubMed PMID: 25552285.

45. Southwell DG, Garcia PA, Berger MS, Barbaro NM, Chang EF. Long-term seizure control outcomes after resection of gangliogliomas. Neurosurgery. 2012 Jun;70(6):1406-13; discussion 13-4. PubMed PMID: 22353798.

46. Gonzalez-Darder JM, Gonzalez-Lopez P, Talamantes F, Quilis V, Cortes V, Garcia-March G, et al. Multimodal navigation in the functional microsurgical resection of intrinsic brain tumors located in eloquent motor areas: role of tractography. Neurosurgical focus. 2010 Feb;28(2):E5. PubMed PMID: 20121440

47. Chang EF, Wang DD, Perry DW, Barbaro NM, Berger MS. Homotopic organization of essential language sites in right and bilateral cerebral hemispheric dominance. Journal of neurosurgery. 2011 Apr;114(4):893-902. PubMed PMID: 21235314.

\section{Figures}

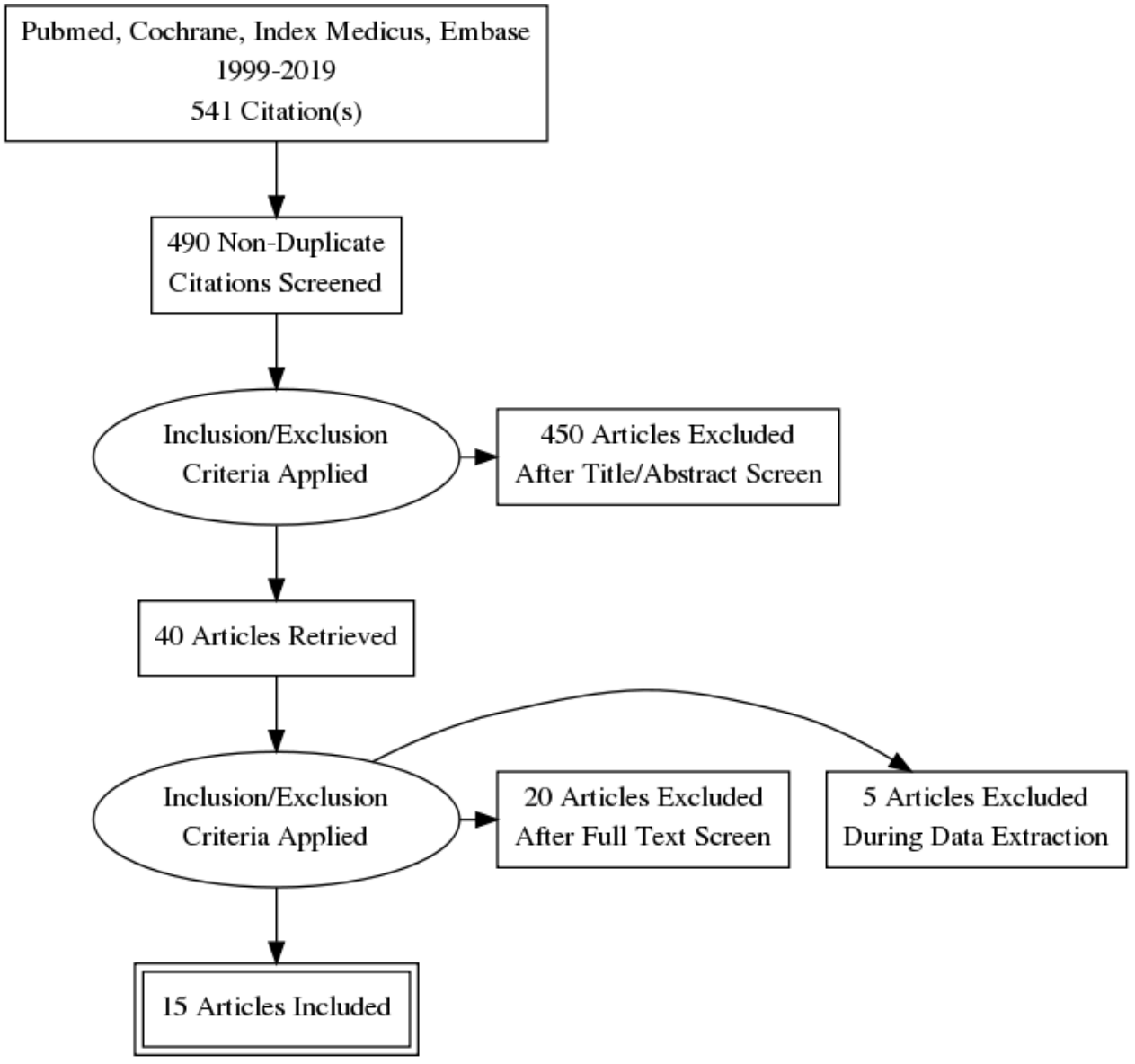

Figure 1

Prisma flow diagram describing the study selection 
Overview of most frequent use of iMRi in epilepsy surgery depending on anatomopathological diagnosis for lesional

(upper part) or non-lesional (lower part) epilepsy
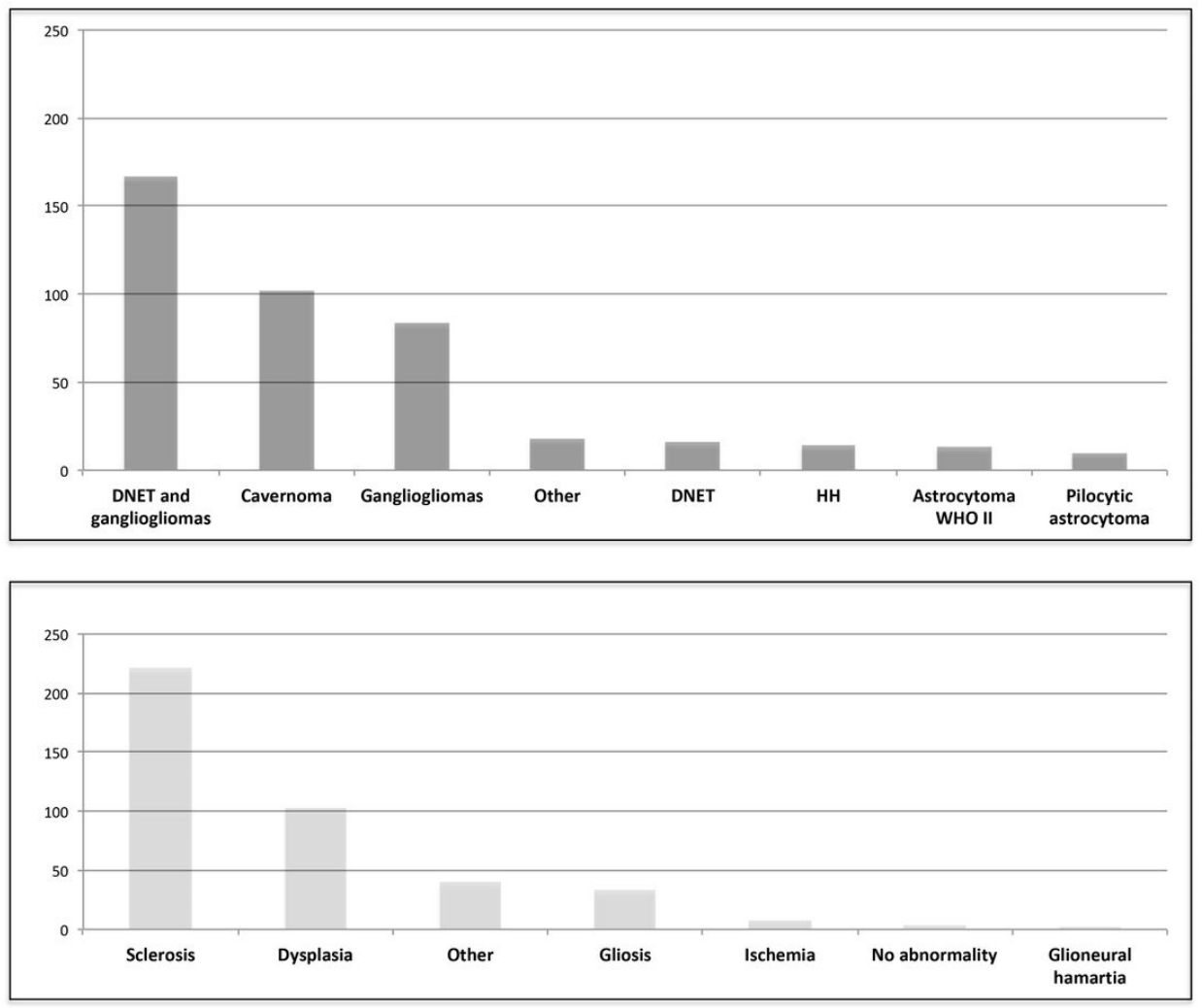

Figure 2

Overview of the most frequent use of iMRI in epilepsy surgery depending on the anatomopathological diagnosis for lesional (upper part) and non-lesional (lower part) epilepsy 

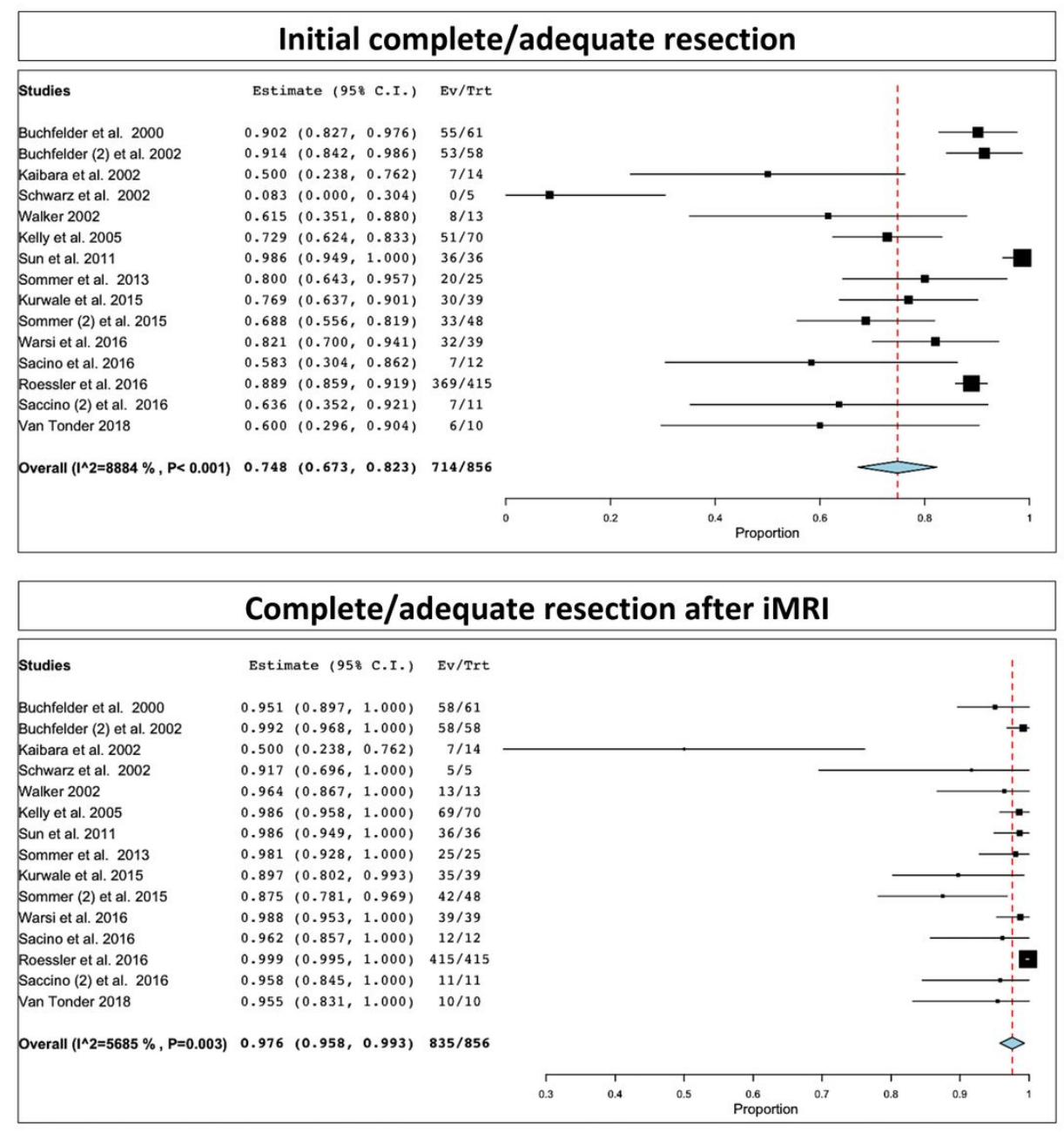

\section{Figure 3}

Initial complete/adequate resection rates before intraoperative MRI use (upper part); complete/adequate resection rates after intraoperative MRI use (lower part) 


\section{Engel class I as outcome at last follow-up}

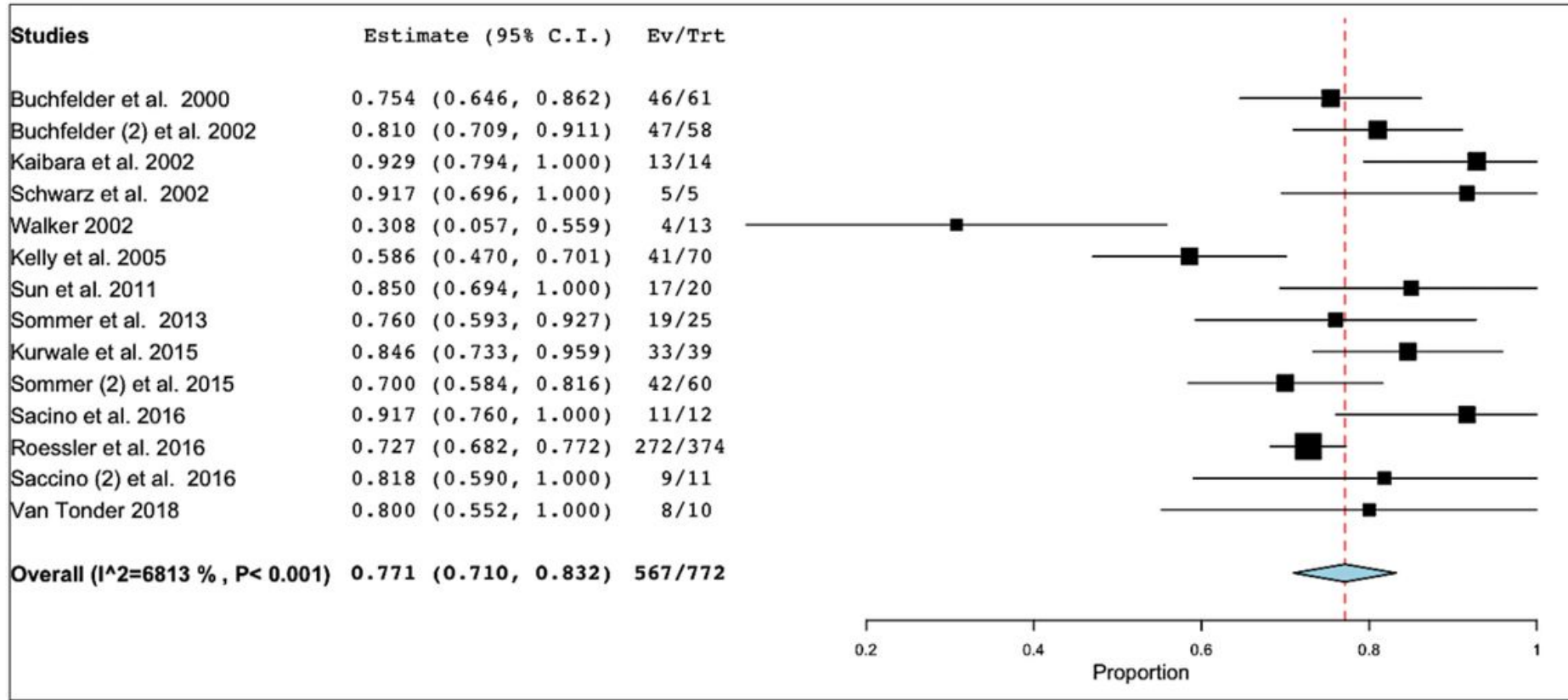

Figure 4

Engel class I as outcome at last follow-up

\section{Supplementary Files}

This is a list of supplementary files associated with this preprint. Click to download.

- SupplementaryMaterielNLEonly.pdf 